\title{
OPTIMAL PATH IN HYBRID DYNAMIC SYSTEMS
}

\author{
S. Esteva, P.Ll. de la Rosa, J. Aguilar-Martin * \\ Dept. Electronic Informatic and Automatic Univ. of Girona \\ *Groupe AS, LAAS/CNRS Toulouse
}

\begin{abstract}
This work treats the analysis and control of hybrid systems using hybrid controllers. The goal is to find the optimal solution according to a performance measure and verifying the solution in order to assure or avoid some misbehaviour. Abstraction is applied over the events generated by continuous signals in order to modify the set of controllable and observable events, and apply Ramadge and Wonham theory of Discrete Event Systems. Optimisation procedure applied over reachability ways obtains the optimal solution and the continuous signals to apply at the system in order to obtain the best performance measure. Copyright ${ }^{\odot} 2000$ IFAC
\end{abstract}

Keywords : Hybrid, Reachability, Optimization.

\section{INTRODUCTION}

Hybrid systems are dynamic systems with continuous signals and discrete events, which can be interpreted by states with continuous dynamic behaviour approximated by ordinary differential equations. This work treat a particular class of the hybrid dynamics systems, systems represented with linear stable continuous dynamics in each state.

In the hybrid systems control, the goal is to find an admissible and controllable path between the initial point in the starting state and the goals End conditions. A controllable system, is a system, which enable to drive through the optimal path towards the goal, with possible uncontrollable events in the path.

We present a methodology to solve the problem to find the optimal path in respect a performance measure, which use different software util in order to obtain: a continuous abstraction, a controllable paths and the optimal control for a class of the hybrid dynamic systems.

Ramadge \& Wonham's theory (Wonham 87) along with the TCT software gives the Supremal Controllable Sublanguage of the legal language generated for a Discrete Event System (DES). Continuous abstraction transforms predicates over continuous variables into controllable events, and modifies the set of uncontrollable and unobservable discrete events.

The verification of the hybrid system assures reachability for the solution, safety and liveness for the system. This is useful to synthetise safety controllers (Puri and Varaiya 95).

Verification of the Supremal solutions determines the right paths that control the system, reaching all the predicates in the transition states and the goal's end conditions.

An optimisation method decides the optimal way and the continuous control to minimise the applied performance measure.

\section{REACHABLE SETS}

Let it be the following SISO linear system (1)

$$
\begin{aligned}
& \dot{X}=A X+B U \\
& Y=C X
\end{aligned}
$$

With $\mathrm{X} \in \mathrm{R}^{2}$ and $\mathrm{A}$ stable, and $\mathrm{U}$ whiting limit bounds $|\mathrm{U}|<\mathrm{U}_{\max }$

The system evolves in time, to the stationary solution for the stable systems when stabilisation time has been reached. The union of the maximum limits on the different trajectories gives the reach set of the system with $\mathrm{U}(\mathrm{t})$ in an interval range.

The reach set of the system with $\mathrm{C}=[0 \mathrm{k}]$ is initial condition independent:

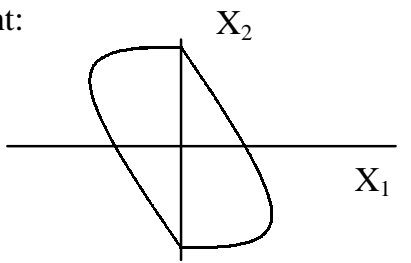

Figure 1 : Reachability set of a state

With $\mathrm{X}_{1}$ and $\mathrm{X}_{2}$ limits as the maximum response to the Bang-Bang control. Inside of the region there are all of the reachable states with finite time for this system (1). 
Reachability analysis of the system must consider two possible situations, either reaching the bound limits in the transition conditions or reaching the goal.

In the first case, the intermediate state reachability analysis determines if the system crosses the bound limits of the linear predicate (2), which leads the system to the next state. Linear predicates are compound by linear inequalities with first order $\operatorname{logics}(*)$. With $K_{i} \in \mathrm{R}$.

$$
\begin{aligned}
& k_{1} X_{0} \geq k_{1} * k_{2} X_{2} \geq k_{3} \\
& X_{i}=\left(k_{4}, k_{5}\right) \\
& X_{f}=\left(k_{6}, k_{7}\right)
\end{aligned}
$$

Theorem 1: If part of the reachable set (figure 1) is superposed with this region (2), then there exists a finite signal control $\mathrm{U} \in\left[\mathrm{U}_{\min }, \mathrm{U}_{\max }\right]$ which activates the condition transition (2).

In the second case, the point's reachability in the End State is to be defined as the ability to reach the goal $\mathrm{X}_{\mathrm{f}}(3)$ on the hybrid system.

Theorem 2: If the end condition is inside of the reachable set (figure1), then it exists a finite signal control $\mathrm{U} \in\left[\mathrm{U}_{\min }, \mathrm{U}_{\max }\right]$ which translates the system (1) from some initial condition in the state to the goal $\mathrm{X}_{\mathrm{f}}(3)$ of the state space.

Using the combination of this two properties the verification procedure assures the reachability of the $\mathrm{X}_{\mathrm{f}}(3)$ End point for a hybrid system, starting at another state with initial condition $\mathrm{X}_{\mathrm{i}}(3)$. We call this a Reachable Way (RchW). Safety property is assured if there are no solution (RchW) to any non-permitted state.

\section{CONTINUOUS ABSTRACTION}

To apply the RW theory (Wonham 87) of discrete event systems on a hybrid system, we must abstract the continuous signals and project the events generated for these signals, to obtain observable and controllable sets of events.

Continuous signals produce in to the system events when this crosses the limit (2). If this event is deterministic they can be abstracted. The problem is to determine the controllability of this event, in order to assign this to the corresponding set, $\left\{\Sigma_{c}, \Sigma_{u c}, \Sigma_{o}, \Sigma_{u o}\right\}$, controllable, uncontrollable, observable and unobservable set of events.

The set of uncontrollable events has to be projected to an other set, by eliminating the uncontrollable events that are indirectly controllable, or continuous reachable, by the continuous control $\mathrm{U}(\mathrm{t})$.

$$
\sum_{u c}=P\left(\sum_{u c}^{\prime}\right)
$$

These events are those that the region generated by the linear predicate (2) traverses the reachable set.

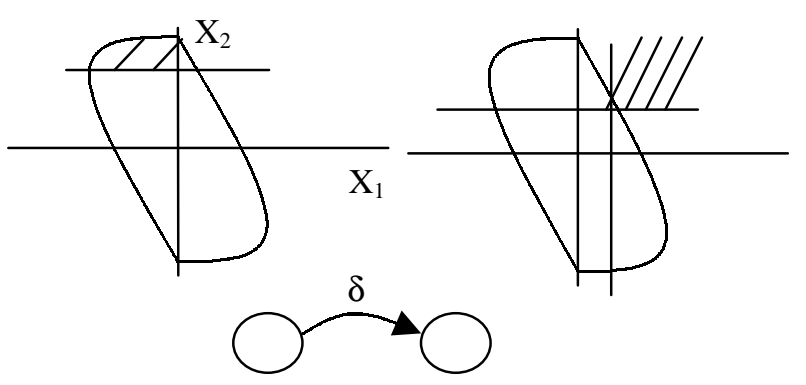

Figure 2: predicates $\delta: \mathrm{X}_{2}>\mathrm{K}_{1}$ and $\mathrm{X}_{1}>\mathrm{K}_{1} \wedge \mathrm{X}_{2}>\mathrm{K}_{2}$

States with more tan one uncontrollable transition can change behavior of the graph, when the events can be converted in to controllable events, this transitions becomes deterministic.

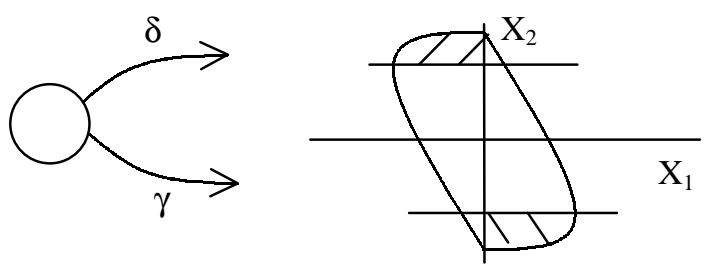

Figure 3: predicates $\mathrm{X}_{2}>\mathrm{K}_{1}$ and $\mathrm{X}_{2}<\mathrm{K}_{2}$

The projection over the unobservable events, generated by the discrete or continuous signals, eliminating the set of observable events by the continuous signals analysis (Lemmon and Antsaklis 94).

$$
\sum_{u o}=P\left(\sum_{u o}^{\prime}\right)
$$

Unobservable events detected when the state is out of the reach set of the model, indicating the use of the other reach set, this means other model, other state. Not observable in continuous sense because is not possible to determine the state change instant by identification methods. Otherwise is observable in discrete sense if the state crosses the reach bounds.

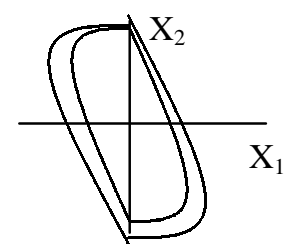

Figure 4: Observability of the state

The transition is true unobservable if the event is not presented and is unidentifiable by continuous identification, and doesn't go out of the reach set of the current model. 
At the same time the uncontrollable events that has been eliminated are added to the set of observable events. A similar procedure will be applied to the unobservable events.

$$
\sum_{c} \cap \sum_{u c}=0, \quad \sum_{o} \cap \sum_{u o}=0
$$

These sets are disjoint (6)

The RW theory (Wonham 87) defines a Controllable Sublanguage, with respect to a language that is composed of erased unobservable events:

$$
\bar{K} \Sigma_{u} \cap L \subseteq \bar{K}
$$

This means that uncontrollable events present in some prefix of the Controllable Sublanguage $\bar{K}$, permitted for the language $L$ of the system, is part of the Controllable Sublanguage. And the Supremal Controllable Sublanguage is the union of the different Controllable Sublanguages.

\section{FAULTS}

A fault is said to be detectable in discrete sense, if exist a transition in the system model that leads to detection in a finite number of steps. And is detectable in continuous sense if the identification method determines a failure state.

Fault is isolable in discrete sense if exists a transition, which gives different behavior or next states for different faults. And is isolable in continuous sense if the identification method determines a unique failure state (Larson 99).

Unobservable events may be failure events or other events that cause changes in the state system not recorded by sensors.

The proceed to fault detection is the next:

1) The continuous signal doesn't progress towards the transition

2) Wait the discrete event to indicate the failure in the system.

3) Analysis of the continuous signals: model identification.

4) Representation the state in the reachable sets.

These four steps allow detect a wide range of the faults. Fault non-identifiables in the steps 1-3, can be detected if the representation of the state (1) is out of the reachable set of the current state.

\section{OPTIMISATION}

To find the optimal solution of RchW to evolve the system from $X_{i}$, to $X_{f}$ (3) going trough different states minimising the performance measure, optimisation methods (Kirk 70) are applied over the set of state sequences of RchW.

In minimum time problem the performance measure is:

$$
J=t_{f}-t_{0}=\int_{t_{0}}^{t_{f}} d t
$$

With $t_{f}$ the first instant of time when $x(t)$ intersects the target set in the end state. Bellman's principle of optimality is applicable in problems that don't present interaction between lateral states.

$$
V^{*}(x)=\min _{p \in \Pi}\left\{g(x, p)+V^{*}\left(x^{\prime}(x, p)\right)\right\}
$$

Where $\Pi$ is the set of states and $g(x, p)$ is the cost of local state, and $\mathrm{V}^{*}\left(\mathrm{x}^{\prime}\right)$ the optimal cost of the rest.

In the transitions defined for $\mathrm{Y}$ limits, the $\dot{Y}$ in the limit ant the signal control $U(t)$ are calculated by dynamic programming (DP) in order to minimise the global time cost. The problem is combined of tree parts, the first is the cost of the first model to approach the system to the limit, the second part is the first model to cross the limit, and the third part is the second model to go through the new limit. The signal applied $U(t)$ in each part is the Bang-Bang (Kirk 70) control in order to optimise the performance measure. In this sense the global optimisation is obtained as the local optimisation of the collateral states.

The interaction between states prohibits the use of local state optimisation. The solution is obtained recursively taken into account the collateral pairs of states until they converges (Esteva 98).

\section{EXAMPLE:}

A simple example to show the possibilities of this method is shown next. Results are not very interesting to the simplicity of the problem.

Let us consider a car with the following speed model, in $\mathrm{Km} / \mathrm{h}$ :

$$
\begin{gathered}
A=\left[\begin{array}{cc}
-.7-G^{\wedge 3} & -1 \\
1 & 0
\end{array}\right] \quad B=\left[\begin{array}{ll}
1 & 0
\end{array}\right] \\
C=\left[\begin{array}{cc}
0 & 35 * G
\end{array}\right]
\end{gathered}
$$

With $U$ restricted to $[0,1]$ and $G$ the gear number.

Determine the RchW and the optimal control, to drive the vehicle as fast as possible from stop (initial state) to end conditions which are defined by a speed 
of $90 \mathrm{Km} / \mathrm{h}$ and acceleration of $.15 \mathrm{~m} / \mathrm{s}$, which is the maximum speed to safety take the curve.

\section{1) The first step is to define the graph evolution:}

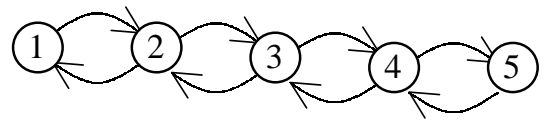

2) Second step is to determine the transition conditions reachability.

The state transitions are triggered in order to maximize the acceleration, they are actives when the next state presents a higher acceleration.

1 to 2 condition transitions: when speed $\geq 28 \mathrm{~km} / \mathrm{h}$ and acceleration $\geq 1 \mathrm{~m} / \mathrm{s}$

2 to 3 condition transitions: when speed $\geq 57 \mathrm{~km} / \mathrm{h}$ and acceleration $\geq .4 \mathrm{~m} / \mathrm{s}$

3 to 4 condition transitions: when speed $\geq 82 \mathrm{~km} / \mathrm{h}$ and acceleration $\geq .24 \mathrm{~m} / \mathrm{s}$

4 to 5 condition transitions: when speed $\geq 115 \mathrm{~km} / \mathrm{h}$ and acceleration $\geq .1 \mathrm{~m} / \mathrm{s}$

The state transitions' reachability conditions are the following:
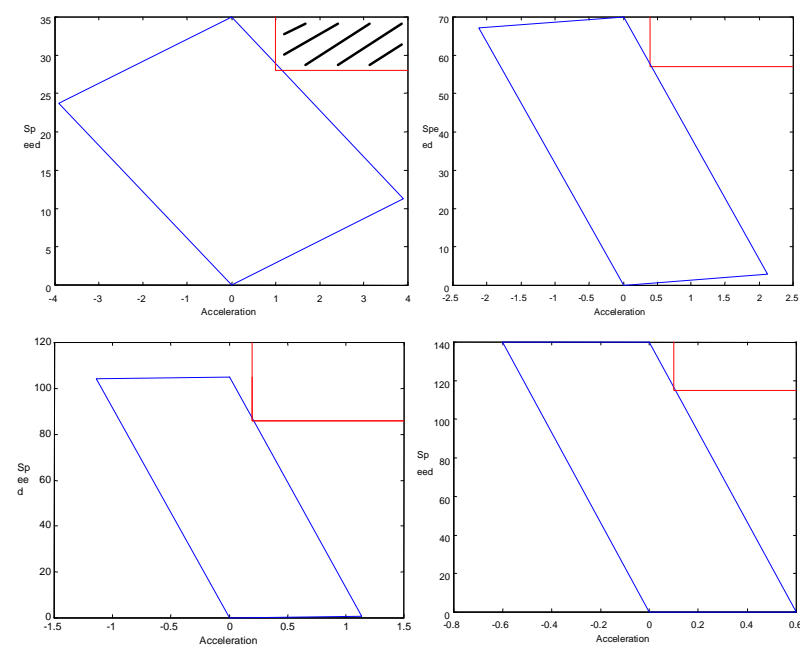

Figure 5.1: Transition conditions reachability for $1^{\text {st }}$, $2^{\text {nd }}, 3^{\text {rd }}$ and $4^{\text {th }}$ gear

The graph shows that transitions, 1 to 2,2 to 3 , and 3 to 4 , are reachable.

The reachabilty set in finite time is given into these limits:

1: $3.9 \mathrm{~m} / \mathrm{s}$ at $11.25 \mathrm{~km} / \mathrm{h},-3.9 \mathrm{~m} / \mathrm{s}$ at $23.5 \mathrm{~km} / \mathrm{h}$, and maximum $35 \mathrm{~km} / \mathrm{s}$.

2: $2.12 \mathrm{~m} / \mathrm{s}$ at $2.8 \mathrm{~km} / \mathrm{h},-2.12 \mathrm{~m} / \mathrm{s}$ at $67.2 \mathrm{~km} / \mathrm{h}$, and maximum $70 \mathrm{~km} / \mathrm{h}$.

3: $1.14 \mathrm{~m} / \mathrm{s}$ at $.66 \mathrm{~km} / \mathrm{h}$ and $-1.14 \mathrm{~m} / \mathrm{s}$ at $104 \mathrm{~km} / \mathrm{h}$ and maximum $105 \mathrm{~km} / \mathrm{h}$.
4: $.6 \mathrm{~m} / \mathrm{s}$ at $.18 \mathrm{~km} / \mathrm{h}$ and $-.6 \mathrm{~m} / \mathrm{s}$ at $139 \mathrm{~km} / \mathrm{h}$ and maximum $140 \mathrm{~km} / \mathrm{h}$.

5: $.38 \mathrm{~m} / \mathrm{s}$ at $.12 \mathrm{~km} / \mathrm{h}$ and $-.38 \mathrm{~m} / \mathrm{s}$ at $174 \mathrm{~km} / \mathrm{h}$ and maximum $175 \mathrm{~km} / \mathrm{h}$.

The end condition's reachability, $90 \mathrm{~km} / \mathrm{h}$ of speed and $.15 \mathrm{~m} / \mathrm{s}$ of acceleration, can be seen in the figure:

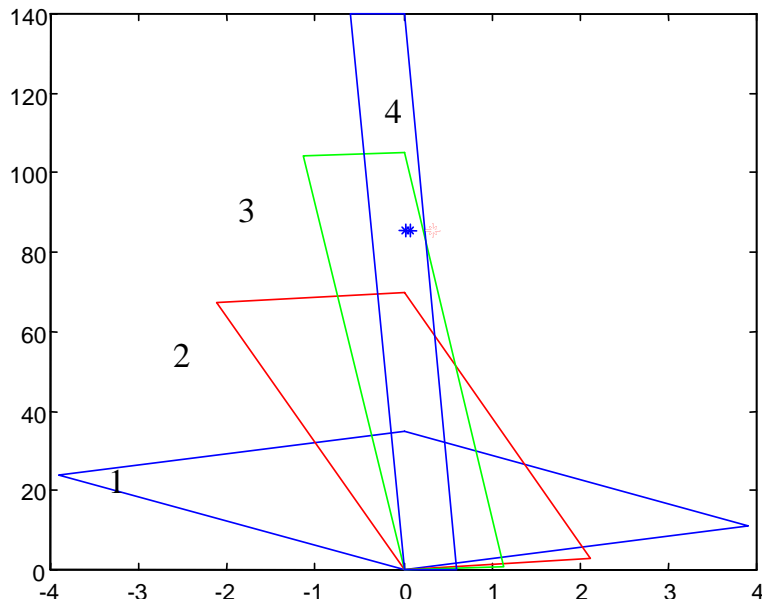

Figure 5.2 : End conditions reachability.

In the case of multiple solution, the end point's reachability is obtained by evaluating the different solutions in order to determine the optimum. When the models have similar rising times, the optimal state will be the one having its reach set limits most distant to the end point.

3) Apply the abstraction over continuous signals, to obtain the set of observable and controllable events.

The events generated by the continuous signals are reachables, this implies to pertain at the set of controllable events:

$\Sigma_{c}^{\prime}=\{$ transition: $1-2,2-3,3-4,4-5\}$

4) Step to obtain controllable languages.

TCT (Thistle 94) yields the Supremal Controllable Sublanguages to drive the system from state 1 to state $\mathrm{X}(2,3,4$, or 5$)$ :

Once the reachability criteria of the states are met, the path trough those states will determine the RchW.

Two possible RchW : 1 -> 2 -> 3 and 1 -> 2 -> 3 -> 4.

The $5^{\text {th }}$ gear doesn't appears in the solution because the transition $4->5$ is not reachable before the End point.

The two possibilities must be analysed to determine the fastest possibility. In this case, without delays in the transitions of the states, the fastest one is $1->2$ $>3->4$, because the $4^{\text {th }}$ gear is faster that the $3^{\text {th }}$ one when its use is possible. 
5) Optimization method calculates the control signal $U(t)$, which concludes that the minimum time is 40 seg.

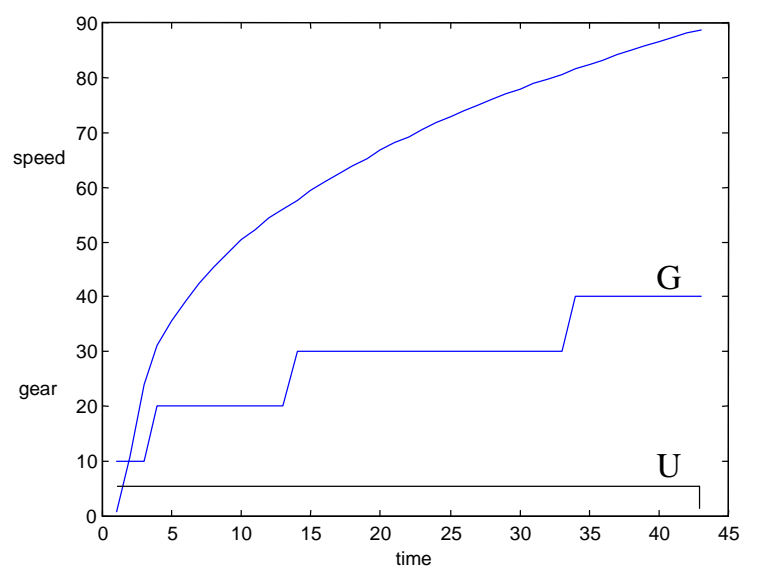

Figure 5.3: Continuous signal $\mathrm{U}(\mathrm{t})$, and discrete $\mathrm{G}$

\section{6) Fault detection.}

In cases when the fault can not be detected by inspection of the continuous signals, for example when the wind perturbation doesn't allow identify the exact model. Faults can be detected by inspection of the reachable sets for automatic car.

When the state is out of the current reachable set. This method determines that the actual state (gear) is bigger than the expected state when the state crosses the limits of the reach set for the side of the velocity, and otherwise in the side of acceleration, the actual state is a fewer gear.

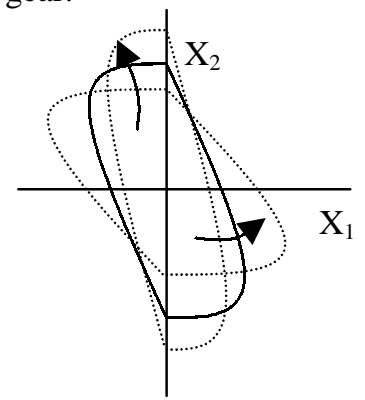

Figure 5.4: Observable faults

\section{CONCLUSION}

This paper shows one methodology to work with hybrid systems in order to control and minimise performance measures. Integrators can be used in this methodology for robot path planning, so can be dynamic scenarios to assure safe paths, that is, without collisions.

To automate the procedure is difficult do to a particular analysis must be carried out for each problem, and besides, different optimisation methods can be applied. Now work is being done in this sense to generate high level code, which allows the modelisation (Esteva 99) and verification of the hybrid systems.

\section{REFERENCES}

Esteva. S. "Model.litzacio de Sistemes Hibrids". Univ. de Girona Treball de recerca 98-43 EIA

Esteva. S.”Modelling Hybrid Systems using State Space regime Decomposition". STAR'99 Barcelona, Univ. Politècnica de Catalunya.

Larson M. "Behavioural and Structural Model Based Approaches to Discrete Diagnoses" Ph.D. dissertation, Dept Elec. Eng. Linkoping Univ. Sweden 1999

Lemmon M.D. P.J. Antsaklis. "Event Identification in Hybrid Control Systems". 32 Conf. Decision and Control 1994.

Puri A. and P. Varaiya. "Driving Safely in Smart Cars". California PATH Research Report UCBITS-PRR-95-24. August 1995. Also in Proc. American Control Conference: 3597-3599, 1995.

Thistle J.G., and W.M Wonham. "Supervision of infinite behaviour of discrete-event systems", SIAM J. on Control and Optimization 32 (4), July 1994, 1098-1113.

Kirk D. “Optimal Control Theory” University Book Publishing Company 1970

Wonham W., and P.Ramadge. "Supervisory Control of a Class of Discrete Events Processes". SIAM J Control Optim. 25-1.1987 